\title{
Fanti S, Farsad M, Mansi L (eds): Atlas of PET/CT: A quick guide to image interpretation
}

\author{
First edition, Springer-Verlag, Berlin Heidelberg, 2009, ISBN: 978-3-540-77771-7
}

\section{Zerizer • A. Al-Nahhas}

Published online: 11 March 2009

(C) Springer-Verlag 2009

The use of PET/CT is increasingly becoming routine in many institutions, particularly in the field of oncology. There has also been a surge in the demand for PET/CT case-based reviews and atlases to fulfil the increasing need for educational and training reviews that can aid practitioners in image interpretation.

The atlas currently being reviewed aims at a broad spectrum of physicians, ranging from technicians, junior radiology and nuclear medicine trainees, to qualified consultants. The book is user-friendly with a clear layout that divides its 288 pages into two main sections.

The first section illustrates normal and benign findings of PET/CT, highlighting common and rare pitfalls that the practitioner needs to be aware of whilst interpreting the studies. It is divided into three chapters. The first chapter discusses the normal distribution of FDG in the body illustrated with clinical cases and technical aspects. The second chapter highlights the importance of contrastenhanced PET/CT in the staging of certain tumours. The third chapter describes the pitfalls, based on anatomical locations, that can reduce the sensitivity and specificity of $\mathrm{PET} / \mathrm{CT}$.

The second section contains more than 500 images of pathology on PET/CT illustrated as black-and-white CT and PET images as well as fusion images. This is cleverly presented as case-based reviews that the reader can utilize as problem-solving tools. This second section (chapter 4) reviews the different cancers with a case-based practical approach that can be easily applied clinically. The images are clear, relevant and well annotated with arrows and legends. The cases emphasize the role of PET/CT in making the diagnosis, staging, follow-up and assessment of recurrence. The cases are also extremely contemporary with an emphasis on some innovative techniques such as the use of ${ }^{11} \mathrm{C}-\mathrm{CHOL} \mathrm{PET} / \mathrm{CT}$ in prostate cancer, and also the usefulness of PET/CT in debatable areas such as melanoma and sarcomas is reviewed.

Overall the atlas provides a concise but at the same time comprehensive aid to the interpretation of a variety of pathological processes on $\mathrm{PET} / \mathrm{CT}$ encountered in routine clinical practice.

A major strength of this book is the take-home messages presented at the end of each page as teaching points in highlighted boxes. This is a fantastic aide-mémoire for the practitioner.

Although the use of PET/CT in oncology is extensively reviewed in the atlas, other applications of PET/CT such as in neuroimaging are not covered. This was perhaps beyond the scope of this book.

Overall, the authors present us with an efficient, userfriendly, well-written and illustrated atlas that any practitioner can utilize in their daily practice in the interpretation of PET/ CT images in oncology.

I. Zerizer $\cdot$ A. Al-Nahhas $(\bowtie)$

Imperial College Healthcare Trust,

London, UK

e-mail: aal-nahhas@hhnt.org 